\title{
Social-Ecological Barriers to Student-parents' Academic Success: A Systematic Review
}

\author{
Kobi V. Ajayi ${ }^{1,2,3^{*}}$, Gloria Odonkor ${ }^{4}$, Sonya Panjwani ${ }^{1,2}$, Oluwafemi Aremu ${ }^{5}$, Whitney Garney ${ }^{1,2}$, \\ and Lisako J. Mckyer ${ }^{6}$ \\ ${ }^{1}$ Department of Health and Kinesiology, Texas A\&M University \\ ${ }^{2}$ Laboratory for Community Health Evaluation and Systems Science (CHESS), Texas A\&M \\ University \\ ${ }^{3}$ Education, Direction, Empowerment, \& Nurturing (EDEN) Foundation, Nigeria \\ ${ }^{4}$ Pharmaceutical Sciences, College of Pharmacy, University of Texas, Austin \\ ${ }^{5}$ Clinical Department, Defense Industries Corporation of Nigeria \\ ${ }^{6}$ School of Public Health, Texas A\&M University \\ *Corresponding Author \\ Kobi V. Ajayi MPH, MBA \\ Department of Health and Kinesiology, Texas A\&M University, College Station, TX 77843. \\ Email: omo_debare@tamu.edu
}




\begin{abstract}
Students who are parents in higher education (HE) in the United States encounter competing demands and challenges while navigating multiple roles as parents, students, and employees. These challenges are multifaceted and can hinder their degree attainment. Using the socioecological model, we systematically summarized existing literature published between 2009 2020 to understand the experiences and predictors of student-parents academic outcomes in HE. The reviewed articles included students from two-year and four-year institutions (community college, undergraduates, and graduate students). The analysis revealed that the constraints to student-parents academic success are a system-level problem. Most of the barriers and facilitators identified were related to structural policies adopted by HE. This study concludes with recommendations for future research, policymakers, and the school ecosystem.
\end{abstract}

Keywords: Parents; Higher institutions; Universities; Academic success; Students 


\section{Introduction}

The proportion of students raising children in higher education (HE) has persistently increased in the past decade in the United States (U.S.). As of the 2015 -2016 school year, an estimated 4.3 million or 22 percent of all undergraduate students in (U.S. United States Government Accountability Office, 2019) were raising children. However, mothers account for $70 \%$ of the overall student-parent population, with a greater proportion of mothers being single (62\%), whereas $61 \%$ of fathers are married (Cruse et al., 2019; United States Government Accountability Office, 2019).

Despite the increasing numbers, student-parents experience marked challenges to their educational attainment, which leads to higher dropout rates than their non-student-parent counterparts. The United States Government Accountability Office reports that $52 \%$ of studentparent dropped out of school without earning a degree than $32 \%$ of students without children. Furthermore, among the student-parent population, $28 \%$ of student-parents who are single parents managed to earn a degree within six years of enrollment (Cruse et al., 2018; United States Government Accountability Office, 2019). However, no single reason explains the high dropout rates among student-parents because the barriers they encounter are multifaceted.

High shares of student-parents are from low-socioeconomic backgrounds and cannot afford tuition costs, quality/affordable childcare, affordable housing, and reliable transportation. Furthermore, time poverty stemming from balancing caregiving demands, school responsibilities, and work requirements continue to be persistent barriers reported in the literature. Another well-cited barrier is the lack/insufficient institutional support targeting student-parent's need in HE (Gault et al., 2014; Cruse et al., 2019). Issues include unclear institutional policies, poor support from faculty and staff, and, importantly, limited on-campus 
childcare centers that do not meet the growing numbers of student-parents. These challenges are similar across institutions (e.g., community colleges, universities, for-or-not-for profit institutions; Cruse et al., 2019; Gault et al., 2014; Wladis et al., 2018). Also, structural barriers such as limited federal and state policies have been reported. For example, the only federal childcare program (Child Care Access Means Parents in School - CCAMPIS) that supports lowincome student-parents does not adequately meet their needs. In the 2016-2017 school year, CCAMPIS provided child care assistance to 4,000 children, but another 4,200 were on a waitlist to receive benefits (United States Government Accountability Office, 2019). These challenges are unique to the student-parent population alone and put them at a high risk of not earning a certificate and poverty. This is a serious concern with intergenerational effects; as such, addressing these barriers cannot be overstated (Ajayi et al., 2021).

Studies show that parental educational status is associated with improved health status, future earnings, labor force participation, economic and financial securities for not just the parents but also their children and society. (US Department of Labor, n.d.; United States Census Bureau, 2018a, 2018b). Considering that education "shapes live [and] is the key to lifting people [and future generations] out of poverty and reducing socioeconomic and political inequalities" (The Lancet Public Health, 2020, p. e361), it is imperative that HE pay heightened attention to the academic success of their most vulnerable student sub-populations. However, despite the utility of systematic synthesis, to date and to the best of our understanding, no systematic reviews exist that methodologically summarize the existing literature on student-parents educational outcomes.

Therefore, the purpose of this study was to systematically synthesize the existing literature reporting the lived experience and academic outcome of student-parents in HE in the 
United States, using the socio-ecological model. Although the previous attempt at synthesizing the literature exists, the review's methodological quality is questionable (Lin, 2016; Osam et al., 2017). In this systematic review, we sought to answer the following research questions: what are the barriers and facilitators to student-parents academic success?

\subsection{Theoretical Framework}

This study utilizes the socio-ecological model (SEM) for health promotion by McLeroy et al. (1988) to understand the multifaceted and systems aspects of student-parents lived experiences while attending HE. The SEM posits that human development and patterns of behavior are influenced by 1) Intrapersonal or individual factors (e.g., knowledge, attitudes, behavior, or skills), 2) Interpersonal factors (e.g., relationships among formal and informal groups and social support networks), 3) Institutional factors (e.g., factors within organizations and entities), 4) Community factors (e.g., the relationship between organizations and institutions), and 5) Public policy (e.g., local, state, and national policies).

SEM addresses complex issues from a broad and multilevel perspective while recognizing that single-level analyses are insufficient to address complex and dynamic problems adequately. SEM has been used widely by researchers across different settings to understand organizational behavior, school system, and health outcomes (Dammhahn \& Kappeler, 2009; Figueiredo \& Pereira, 2011; Garney et al., 2021; Lim \& Hoot, 2015; Oishi et al., 2007; Townsend \& Foster, 2013). Scholars agree on its effectiveness, rigor, and methodological strength to understand and explain broad topics across diverse settings. It is an ideal framework for this study because it allows researchers to understand the multifaceted aspects of a studentparents life and how those factors interrelate with one another. This understanding provides a systems perspective of the problem. A systems perspective acknowledges the multiple elements 
of influence, how they impact the problem, and how they interact. This leads to a holistically understanding and allows researchers to identify leverage points for potential intervention and anticipate unintended consequences of change.

Student-parents' abilities to learn about and navigate barriers (individual level), their relationship with faculty and family members (interpersonal level), HE policies such as childcare subsidies (institutional level), and federal programs such as Child Care Access Means Parents in School Program (CCAMPIS; policy level) influence and interact from an ecological perspective on their academic success. This study seeks to identify multifaceted factors using SEM (Figure 1) to understand better common barriers and potential facilitators to Parenting-Students' academic success in colleges and universities. 


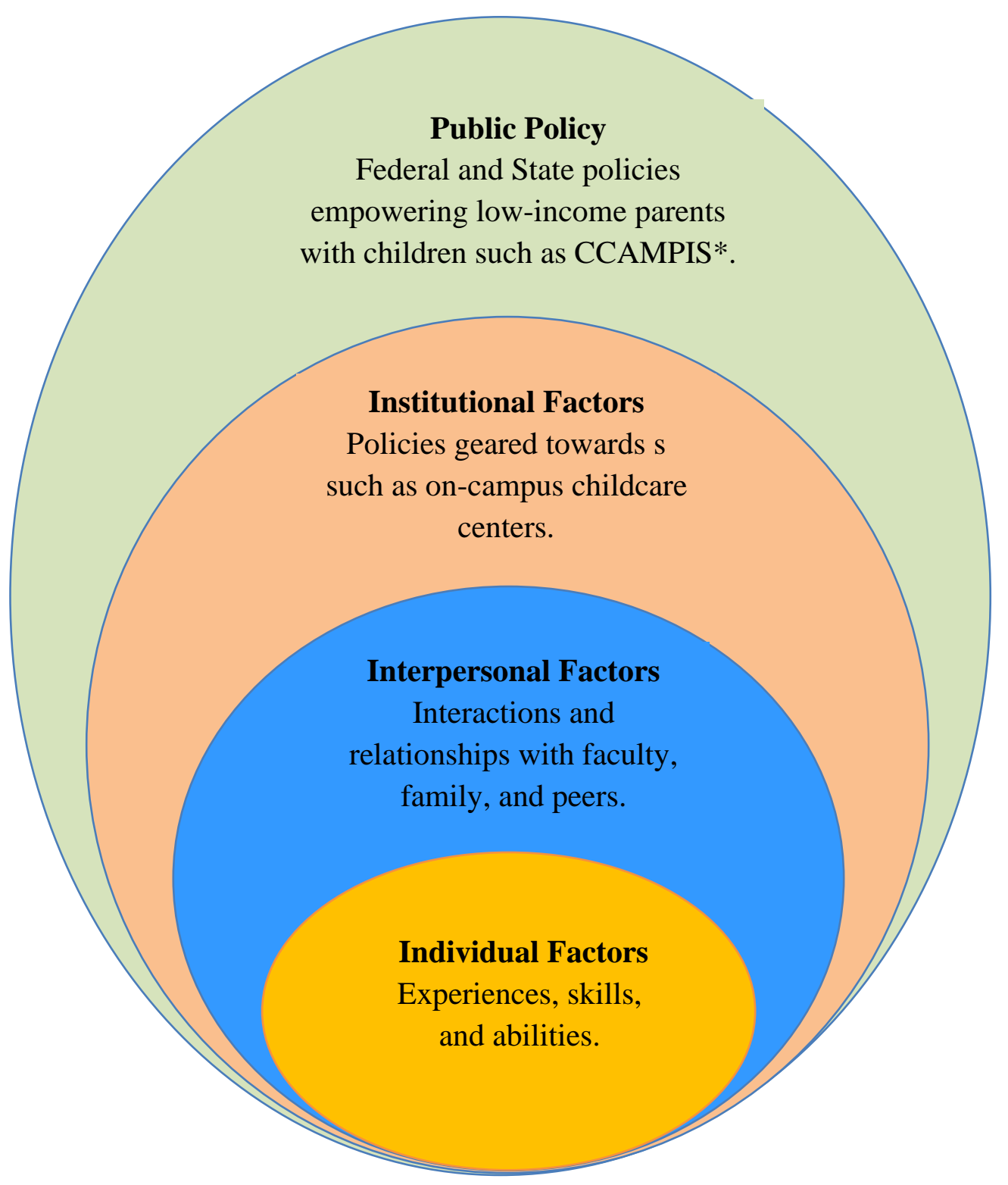

Figure 1. McLeroy and Colleague's socio-ecological framework for health promotion showing the multilevel factors within the individual, interpersonal, institutional, and public-level affecting student-parents academic success in the United States.

* Child Care Access Means Parents in School Program 


\section{Materials and methods}

This systematic review was conducted per the Preferred Reporting Items for systematic review (PRISMA) guideline and checklist. We developed and registered the study protocol with PROSPERO - registration number- CRD42020179912.

\subsection{Search strategy}

A thorough and systematic search was performed of peer-reviewed articles published between 2009 to 2020 from eight major electronic databases: Eric, Education Source, Education Full Text, Education Administration Abstracts, Academic Search Ultimate, APA PsycInfo, Medline, and CINAHL (Ebsco). All search protocols were conducted in tandem with a research reference librarian who is an established expert in health sciences systematic reviews to ensure thoroughness. An initial search was conducted in 2019 and updated in 2020. Additionally, the reference sections of the selected articles were purled to identify articles potentially missed via electronic search. The terms used in the searches included "parents," "mothers," "childrearing," "child raising," "college students," "undergraduate students," "student-parents," "non-traditional students," "students," and "graduate students," in different combinations using the "AND" and “OR" Boolean combinations.

\subsection{Inclusion and exclusion criteria}

We included studies utilizing quantitative and qualitative study designs. The study included students from two-year (e.g., community colleges) and/or four-year institutions (e.g., undergraduates, Masters, Ph.D., and professional students). Part-time or full-time students, if reported but not distant learning students, were included. Finally, studies that examined institutional policies in the context of parenting and or pregnant students were selected. It is noteworthy to state that this review's initial goal was to assess mothers' experiences who may be 
parents or pregnant in HE because they represent a greater proportion of the student-parent population and experience greater challenges to their college attainment than fathers (Cruse et al., 2019). However, a handful of the retrieved articles included fathers who were also students, but their population size was substantially small. Thus, this current Study had fathers and mothers who are parents in the IHE in the US.

Moreover, it was impossible to extract the father's reported academic outcomes separately from the mothers. Given these concerns, we included studies reporting fathers and mothers with children in HE. We excluded articles reporting information about the parents of college students - Figure 2.
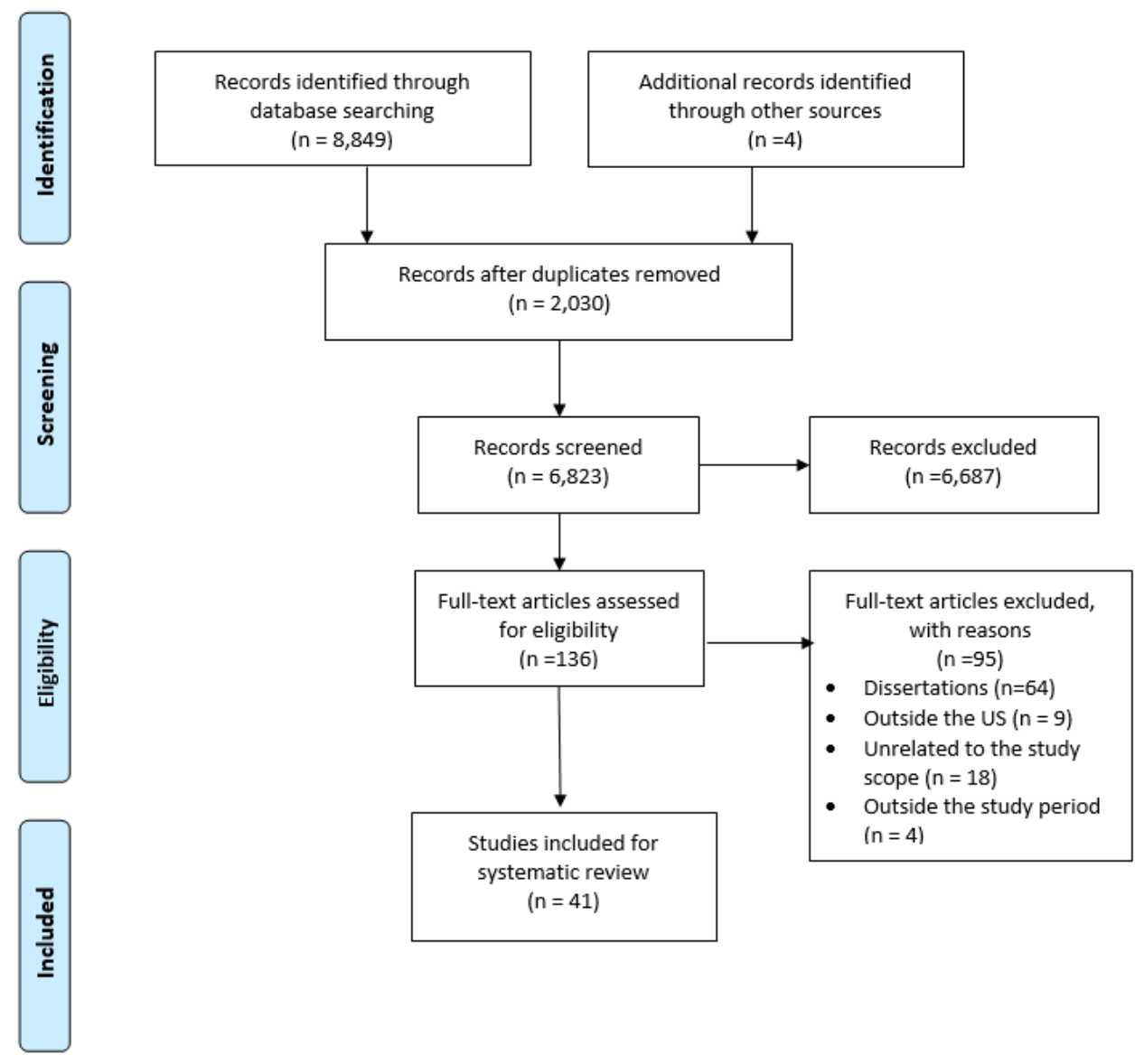

Figure 2. Flow diagram showing the studies included for analysis. 


\subsection{Data extraction and synthesis}

Guided by the socio-ecological model, we extracted studies using the matrix method (Garrard, 2020). Barriers and facilitators associated with student-parents academic outcomes were extracted from each article. Studies retrieved were screened by title and abstract independently by two team members and were subsequently triaged for full-text screening. The team members discussed diverging opinions to establish inter-rater reliability; discordant scores were resolved by consensus. Data points extracted included study design, type of institution, theoretical framework, factors in the SEM levels, and other essential information significant to this reviews' research question and objective. One team member pre-and-pilot-tested the initial form by selecting the first five studies from each study domain (qualitative and quantitative articles). Using a qualitative interpretive framework, the team conducted three iterations of coding, which comprised an initial coding of the articles to label the data, reexamining the initials codes to develop categories, and finally performing a thematic analysis of the data. The codebook was reviewed by a team member who was not involved in the initial screening process to establish validity and reliability. The qualitative analysis enables researchers to inductively and deductively analyze data that is "sensitive to the people and places understudy" while establishing "patterns or themes" (Creswell \& Poth, 2016, p.42).

\subsection{Methodological quality assessment}

We assessed the articles' methodological quality using the Joanna Briggs Institute (JBI) Critical appraisal tool for qualitative and cross-sectional studies, respectively. Two team members independently used the JBI cross-sectional and qualitative assessment tool to rate the quantitative and qualitative articles' methodological quality. Subsequently, the team members 
met to discuss and resolve discrepancies. All articles received a score of a high, low, or unclear rating (high-1, low/unclear-0).

\section{Results}

\subsection{Study characteristics}

A total of 41 articles were included in this review, as seen in Table 1 . The majority $(n=$ $22 ; 53.6 \%)$ utilized a qualitative research methodology, fifteen $(36.5 \%)$ were quantitative studies, and four (9.7\%) used a mixed methodology study design. Thirty-two articles (78\%) included university students (undergraduates, master-level, or doctoral students) or community colleges. Two studies (4.8\%) individually assessed medical students and veterinarians. A small number of articles $(n=11 ; 26.8 \%)$ included fathers, and four articles $(9.7 \%)$ evaluated existing institutional pregnant and parenting students' policies and programs, program directors, administrators, faculty, or staff. Two studies (4.8\%) comprise international students. Of the 41 articles included, only ten $(24.3 \%)$ reported evidence of an existing program or policies targeted at pregnant or parents' students. Overall, twenty-three (56\%) articles used at least one theoretical framework. Extensive details about the study characteristics and methodological quality are reported in the ensuing paragraphs.

In the ensuing paragraphs, we summarize the barriers (Table 2) and facilitators (Table 3) based on their individual, interpersonal, institutional, and policy-level factors. Because an article may report more than one theme or sub-theme, aggregate themes may exceed the total number of articles reviewed $(n=41)$.

Table 1: Study Characteristics of Included Articles 


\begin{tabular}{|c|c|c|c|c|c|c|c|}
\hline ID & Authors & $\begin{array}{l}\text { Study } \\
\text { design }\end{array}$ & $\begin{array}{l}\text { Sample } \\
\text { characteristics, } \\
\text { size }\end{array}$ & $\begin{array}{l}\text { Study method, } \\
\text { analysis }\end{array}$ & $\begin{array}{l}\text { Institution type } \\
\text { and level of } \\
\text { Study }\end{array}$ & $\begin{array}{l}\text { Theoretical } \\
\text { framework }\end{array}$ & $\begin{array}{l}\text { Outcome of } \\
\text { interest }\end{array}$ \\
\hline 1 & $\begin{array}{l}\text { (Yakaboski } \\
\text {,2010) }\end{array}$ & Qualitative & $\begin{array}{l}\text { Single } \\
\text { mothers; } \\
(\mathrm{n}=21)\end{array}$ & $\begin{array}{l}\text { Focus groups; } \\
\text { Needs assessment } \\
\text { survey; thematic } \\
\text { analysis. }\end{array}$ & $\begin{array}{l}\text { University; } \\
\text { Undergraduates }\end{array}$ & $\begin{array}{l}\text { Feminist } \\
\text { epistemologi } \\
\text { cal } \\
\text { framework }\end{array}$ & $\begin{array}{l}\text { College } \\
\text { experiences } \\
\text { and retention }\end{array}$ \\
\hline 2 & $\begin{array}{l}\text { (Wilson \& } \\
\text { Cox, 2011) }\end{array}$ & Qualitative & $\begin{array}{l}\text { Married and } \\
\text { single mothers; } \\
(\mathrm{n}=13)\end{array}$ & $\begin{array}{l}\text { Re-analysis of } \\
\text { interview data, } \\
\text { case study, and } \\
\text { interpretive } \\
\text { phenomenologica } \\
1 \text { analysis }\end{array}$ & $\begin{array}{l}\text { Community } \\
\text { college students }\end{array}$ & $\begin{array}{l}\text { Feminist } \\
\text { critical policy } \\
\text { analysis } \\
\text { theory }\end{array}$ & $\begin{array}{l}\text { Lived } \\
\text { experiences } \\
\text { of single } \\
\text { mothers }\end{array}$ \\
\hline 3 & $\begin{array}{l}\text { (Wilsey, } \\
\text { 2013) }\end{array}$ & $\begin{array}{l}\text { Quantitativ } \\
\mathrm{e},\end{array}$ & $\begin{array}{l}\text { Married and } \\
\text { single mothers; } \\
(\mathrm{n}=95) \text {. }\end{array}$ & $\begin{array}{l}\text { Survey, content } \\
\text { analysis; } \\
\text { Descriptive } \\
\text { statistics }\end{array}$ & $\begin{array}{l}\text { University; } \\
\text { Undergraduates }\end{array}$ & Not specified & $\begin{array}{l}\text { Reasons for } \\
\text { college } \\
\text { enrollment; } \\
\text { How college } \\
\text { enrollment of } \\
\text { mothers } \\
\text { affects } \\
\text { children. }\end{array}$ \\
\hline 4 & $\begin{array}{l}\text { (Deutsch \& } \\
\text { Schmertz, } \\
\text { 2011) }\end{array}$ & $\begin{array}{l}\text { Mixed } \\
\text { method }\end{array}$ & $\begin{array}{l}\text { Female } \\
\text { students, } \\
\text { (Study did not } \\
\text { state the } \\
\text { marital status). } \\
85 \% \text { of the } \\
\text { sample has a } \\
\text { prior } \\
\text { postsecondary } \\
\text { education. } 77 \% \\
\text { have children; } \\
(\mathrm{n}=13)\end{array}$ & Focus groups & $\begin{array}{l}\text { University; } \\
\text { Undergraduates }\end{array}$ & $\begin{array}{l}\text { Feminist } \\
\text { epistemologi } \\
\text { cal theory, } \\
\text { Levin's } \\
\text { conceptualiza } \\
\text { tion } \\
\text { framework. }\end{array}$ & $\begin{array}{l}\text { The } \\
\text { experiences } \\
\text { of adult } \\
\text { women } \\
\text { returning to } \\
\text { school for } \\
\text { their } \\
\text { bachelor's } \\
\text { degree. }\end{array}$ \\
\hline 5 & $\begin{array}{l}\text { (Springer et } \\
\text { al., 2009) }\end{array}$ & $\begin{array}{l}\text { Quantitativ } \\
\mathrm{e},\end{array}$ & $\begin{array}{l}\text { Graduate } \\
\text { Program } \\
\text { Directors; } \\
\mathrm{n}=40\end{array}$ & $\begin{array}{l}\text { Survey; } \\
\text { Descriptive } \\
\text { statistics }\end{array}$ & $\begin{array}{l}\text { University; } \\
\text { Doctoral } \\
\text { students }\end{array}$ & Not specified & $\begin{array}{l}\text { To describe } \\
\text { society's } \\
\text { view of } \\
\text { idealized } \\
\text { mothering } \\
\text { and } \\
\text { academia's }\end{array}$ \\
\hline
\end{tabular}


(Shenoy et Quantitativ Single al., 2016) e;

(Sealey- Qualitative Ruiz, 2013)

(Sallee \& Qualitative Cox, 2019)

(Roy et al. 2018)
Married, single mothers, and fathers, (n =17); School administrators, $(\mathrm{n}=19)$. specify the marital status).

\section{Survey; Community}

Descriptive and bivariate analysis.

parent female

students,

$\mathrm{n}=6,523$ (Study did not

Female parent students, $\mathrm{n}=5$. study; Narrative college analysis/ constant comparative method. college students

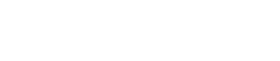

views of an

ideal

graduate

student.

Not specified Difficulties

facing single

parents;

Association

between

single

parenting and

mental health

outcomes

(depression, self-injury, suicide attempt). College re-

Black feminist and entry students. (Study critical race did not state the theory student's degree level).

Community college students document analysis; case studies

Network

analytic

framework

\section{experiences;}

Motherhood;

Effects of reentry on their children's educational choices.

Student parents' college lived experiences.

Not specified Experiences of the transition to motherhood among college students one-on-one interviews; phenomenologica 1 analyses

$\begin{array}{ll}\text { Not specified } & \begin{array}{l}\text { Experiences } \\ \text { of the } \\ \text { transition to } \\ \text { motherhood } \\ \text { among } \\ \text { college } \\ \text { students }\end{array}\end{array}$
mothers, and pregnant students, $(\mathrm{n}=11)$ 


$\begin{array}{lllll}\text { (Ricco et } & \text { Quantitativ } & \text { Married and } & \text { Survey; } & \text { Four-year } \\ \text { al., 2009) } & \text { e; } & \text { single mothers, } & \text { Descriptive, } & \text { university } \\ & & (\mathrm{n}=89) & \text { bivariate, and } & \text { students. (Study } \\ & & & \text { hierarchical } & \text { did not state the } \\ & & \text { analysis. } & \text { student's degree } \\ & & & & \text { level). }\end{array}$

$\begin{array}{ll}\text { Ecological } & \text { college } \\ \text { systems } & \text { mothers' } \\ \text { theory } & \text { student-role } \\ & \text { attitudes to } \\ & \text { their } \\ & \text { parenting } \\ & \text { attitudes and } \\ & \text { to their } \\ & \text { children's } \\ & \text { attitudes } \\ & \text { toward } \\ & \text { school. }\end{array}$

11 (Radey \& Quantitativ Married and Survey: bivariate Cheatham, e; 2013) Longitudin al single mothers, and binomial $(\mathrm{n}=27,269) . * \quad$ logistics regression
University;

Graduate and undergraduate

Intersectional How student ity theory characteristic $s$ influence

FAFSA application rates among low-income, aid-eligible women.

12 (Radey, Quantitativ Married single Survey; e; mothers, Longitudin $\quad(n=2,330)$ al

Descriptive statistics and multilevel logistic regression

13 (Prikhidko \& Haynes, 2018)

4 (Peterson, Qualitative Semi-structured single mothers, Interview;

\section{Community colleges and four-year university.}

University; Doctoral and Master's students 2016) Married and $(\mathrm{n}=8)$

$\begin{array}{ll}\text { Qualitative } & \begin{array}{l}\text { Married and } \\ \text { single mothers, } \\ (\mathrm{n}=8)\end{array}\end{array}$

$\begin{array}{ll}\text { Married, single } & \text { Interview; } \\ \text { mothers, and } & \text { Interpretive } \\ \text { fathers, }(\mathrm{n}=15) & \text { phenomenologica } \\ & 1 \text { analysis }\end{array}$

\section{University;} Community

college students.

\section{Rational Maternal action theory college enrollment rates.}

\begin{tabular}{|c|c|}
\hline $\begin{array}{l}\text { Dissonance } \\
\text { theory; } \\
\text { Constructivis } \\
\text { t theoretical } \\
\text { perspective }\end{array}$ & $\begin{array}{l}\text { Graduate } \\
\text { student } \\
\text { mothers' } \\
\text { experiences } \\
\text { balancing } \\
\text { schooling } \\
\text { and } \\
\text { parenting }\end{array}$ \\
\hline
\end{tabular}

Not specified The lived experiences and retention of nontraditiona 1 community 


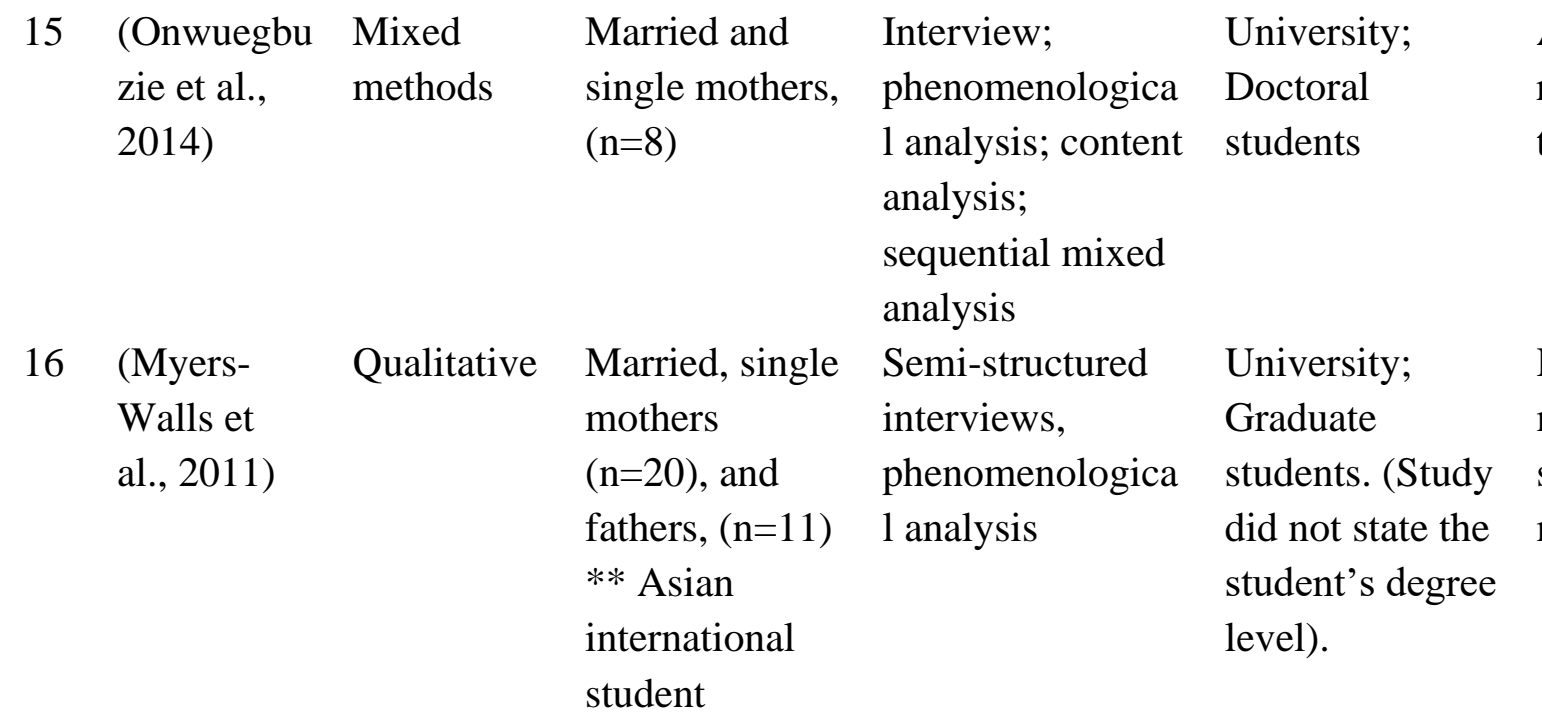

college students with children.

Academic Lived resiliency experiences theory

Hill's ABCX Lived model of experiences stress and management acculturative stressors of Asian international graduatestudent families

17 (Molter et

\begin{tabular}{|c|c|c|}
\hline $\begin{array}{l}\text { Quantitativ } \\
\text { e; cross- } \\
\text { sectional }\end{array}$ & $\begin{array}{l}\text { Websites, } \\
\text { documents ( } \mathrm{n} \\
=26) \text {; School } \\
\text { administrators } \\
\text { from } 30 \\
\text { veterinary } \\
\text { medicine } \\
\text { association, } \\
(\mathrm{n}=20) .\end{array}$ & $\begin{array}{l}\text { Surveys; } \\
\text { document } \\
\text { analysis; } \\
\text { descriptive } \\
\text { analysis; thematic } \\
\text { analysis. }\end{array}$ \\
\hline
\end{tabular}

Veterinary internship or residency program; house officers (postgraduate trainees)

Interviewing; University; Concept-driven Master level students

$\begin{array}{ll}\text { Role conflict } & \text { How } \\ \text { theory } & \text { graduate } \\ \text { student } \\ \text { parents } \\ \text { navigate their } \\ \text { academic, } \\ \text { familial, and } \\ \text { professional } \\ \text { responsibiliti } \\ \text { es. }\end{array}$


19 (Mahaffey Quantitativ Married and Survey; counts $\&$ Gregg, e single mothers, 2015)

$$
(\mathrm{n}=237)
$$

20 (Madden, Qualitative Married, single 2018)

21 (Shepherd \& Nelson, 2012)

$\begin{array}{lll}\text { (E. Lovell } & \text { Quantitativ } & \text { Student } \\ \text { \& Munn, } & \text { e; } & \text { parents, (n } \\ \text { 2017) } & \text { Quasi- } & =70, \text { Study did } \\ & \text { experiment } & \text { not specify } \\ & \text { al } & \text { gender and } \\ & & \text { marital status). } \\ & \text { Non-parenting } \\ & & \text { students, } \\ & & (\mathrm{n}=105) .\end{array}$

(E. D. N. Lovell, 2014)

\section{mothers, and}

pregnant

student, $(\mathrm{n}=17)$ 1 approach
University
students (Study
did not state the
student's degree
level)

Community thematic analysis college students
(1)

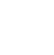
Not specified Mothers' campus service needs and retention barriers Acker's Low-income theory of pregnant and gendered parenting organization student mothers' experiences in community colleges.

University; Cross To Phenomenologica Masters and Doctorate

barriers to understand adult learning

how adults successfully complete their graduate studies despite the barriers they encounter Survey; Bivariate Community Piaget's Learning analysis and college students theory of preferences; cognitive Life meaning development for parenting students vs. non parenting students.

Undergraduates from two-year and four-year campuses. analysis and linear regression models models

Survey; Bivariate e; Quasi- mothers, and experiment fathers, $(n=78)$ al

$\begin{array}{ll}\text { Not specified } & \text { Retention } \\ & \text { needs of } \\ \text { student } \\ \text { parents with } \\ \text { children } \\ \text { below and }\end{array}$


24 (Lobn

25 (Lindsay \& Qualitative Gillum, 2018)

(Lashley \& Qualitative Ollivierre, 2014)

(Plageman Quantitativ \& Sabina, e 2010)

28 (Kulp, 2020)
Married and single mothers, $(\mathrm{n}=23)$.

African international student

\section{Single} mothers, $(\mathrm{n}=10)$.
Semi-structured interviews.

Oral and life

history interviews; Focus groups; content analysis interviews.
Semi-structured interviews; conventional content analysis

Survey;

Descriptive;

correlation Study did not state the marital status) $59.26 \%$ have a spouse or partner; $46.3 \%$ have a child[ren]

$\begin{array}{lll}\text { Quantitativ } & \text { Married and } & \text { Survey; } \\ \text { e; } & \text { single mothers } & \text { Descriptive } \\ \text { Longitudin } & (\mathrm{n}=214), & \text { statistics; } \\ \text { al } & \text { fathers, }(\mathrm{n}= & \text { Sequential } \\ & \text { 248). Total } & \text { logistic regression } \\ & \text { sample } & \end{array}$

University;
Graduate
students (Study
did not state the
student's degree
level).

University;

(ever).

University; Undergraduates

above 6 years old.

Not specified Structural and sociocultural environments of African graduate student mothers Bioecologica Single 1 model of mother human perceptions development. and experiences with campus related services.

University; Not specified Lived Undergraduates experiences of Black undergraduat e college mothers.

Universities; Not specified Family Undergraduates support on college attendance, GPA, and persistence.

$\begin{array}{lll}\text { University; } & \text { Enders' } & \text { tenure-track } \\ \text { Doctoral } & \text { framework } & \text { job outcomes } \\ & \text { integrated } & \text { of graduate } \\ & \text { with } & \text { student } \\ & \text { Kennelly and } & \text { mothers after } \\ & \text { Spalter- } & \text { graduation }\end{array}$


includes non-

parent

students. $(\mathrm{N}=$

2,994).

29 (Kensinger Qualitative \& Minnick, 2018)

\section{0}

(Johnson \& Quantitativ Nussbaum, e; 2012)

31 (Hotez et al., 2020)

Qualitative

Married female students, $n=5$ males, $(n=84$; $60 \%$ have been married \& $30 \%$ are parenting). Non-parent students, $n=94$
Semi-structured interview; thematic analysis
Roth's

framework

University; Gender-role Support and Undergraduates theory and barriers of social capital mothers theory pursuing higher education.

University; Undergraduates Survey;

Descriptive,

MANOVA, and correlation analysis

chas

Achievement

goal theory goals and coping strategies between traditional and

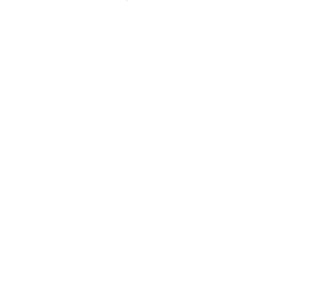

University;

Four-year

institutions.

(Study did not

state the

student's degree

level).

Achievement

nontraditiona 1 students.

Not specified Perspectives, experiences, and priorities of studentparents that may impede or bolster their success in higher education.

32 (Markle, Mixed Married and Survey; University; Descriptive and Undergraduates bivariate analysis; $(\mathrm{n}=320)$, fathers $(n=174)$ Grounded theory, Logistic regression
Role theory Factors influencing persistence among nontraditiona 1 men women undergraduat e students.

Not specified The causes of tension and stress 


\begin{tabular}{|c|c|c|c|c|c|}
\hline 34 & $\begin{array}{l}\text { (Dinour et } \\
\text { al., 2015) }\end{array}$ & Qualitative & $\begin{array}{l}\text { Married and } \\
\text { single mothers, } \\
(\mathrm{n}=11), \text { Staff } \\
(\mathrm{n}=8) \text {, and } \\
\text { Faculty }(\mathrm{n}=13)\end{array}$ & $\begin{array}{l}\text { Semi-structured } \\
\text { interview; content } \\
\text { analysis }\end{array}$ & $\begin{array}{l}\text { University; } \\
\text { Undergraduate } \\
\text { and graduates. }\end{array}$ \\
\hline 35 & $\begin{array}{l}\text { (Crispin \& } \\
\text { Nikolaou, } \\
\text { 2019) }\end{array}$ & $\begin{array}{l}\text { Quantitativ } \\
\mathrm{e}\end{array}$ & $\begin{array}{l}\text { Male and } \\
\text { female parent } \\
\text { and non-parent } \\
\text { students, }(\mathrm{n}= \\
7,161) . * * *\end{array}$ & $\begin{array}{l}\text { Survey; } \\
\text { Descriptive, 24- } \\
\text { hour time diary, } \\
\text { bivariate, linear } \\
\text { regression } \\
\text { models. }\end{array}$ & $\begin{array}{l}\text { Community } \\
\text { college } \\
\text { students, } \\
\text { University } \\
\text { (undergraduates } \\
\text {, masters, and } \\
\text { higher). }\end{array}$ \\
\hline 36 & $\begin{array}{l}\text { (Bye et al., } \\
2017 \text { ) }\end{array}$ & $\begin{array}{l}\text { Quantitativ } \\
\mathrm{e}\end{array}$ & $\begin{array}{l}\text { Male and } \\
\text { female parent } \\
\text { and non-parent } \\
\text { students } \mathrm{n}= \\
\text { 194. *** }\end{array}$ & $\begin{array}{l}\text { Survey; } \\
\text { Descriptive } \\
\text { statistics }\end{array}$ & $\begin{array}{l}\text { Medical } \\
\text { students }\end{array}$ \\
\hline
\end{tabular}

37 (Brown \& Qualitative Married, single Case study; Nichols, 2013)

38 (Bloom, 2009) mothers, Interviews

pregnant, and

fathers $(n=24)$ while

completing a

doctoral

program.

Theory of planned

behavior

.

Behavioral, normative, and control beliefs regarding pumping breast milk on campus between students, staff, and faculty.

Not specified Timeallocation decisions of studentparents.

$\begin{array}{ll}\text { Not specified } & \begin{array}{l}\text { Effects of } \\ \text { pregnancy }\end{array} \\ \text { and } \\ \text { parenthood } \\ \text { on medical } \\ \text { students. }\end{array}$

University; Bourdieau's The policies and programmati $c$ needs of pregnant and parenting students. Undergraduates and graduates theory of social reproduction

Interviews; single mothers, ethnography $(\mathrm{n}=5)$

\section{University:} undergraduates, graduates, and recent graduates
Not specified Contribution $\mathrm{s}$ of personal and educational 


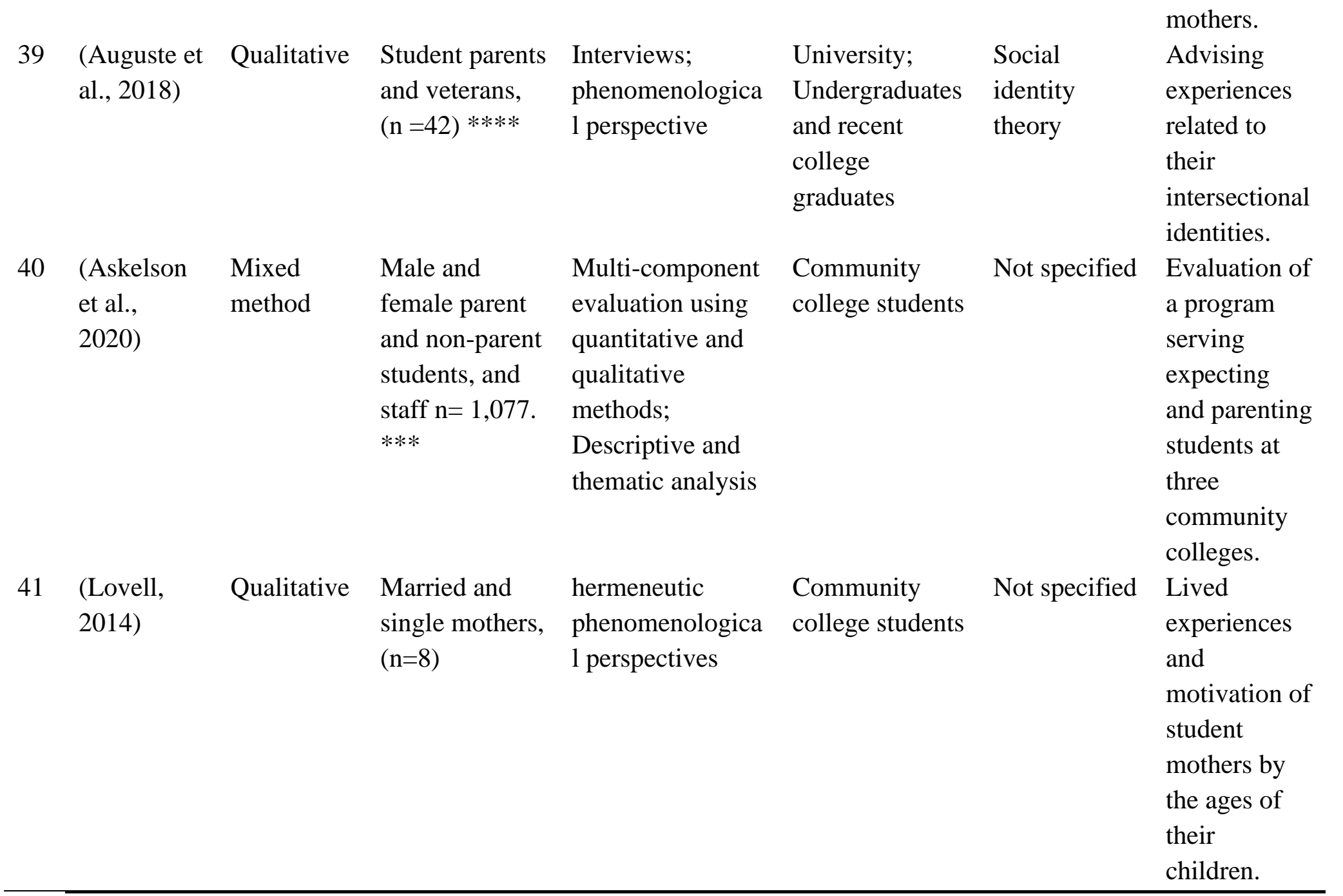

* Females were mothers, veteran (other independent) and other females as a whole (dependent)

** Spouses of students of Asian international students were included

**** Study defined sample as nontraditional students (mothers, veterans, aged 24 or older)

***Study did not report the sample breakdown between parent and non-parents

\subsection{Primary Theme: Barriers to Student-parents' Academic Outcomes}

\subsubsection{Individual-level factors}

The individual-level factors are associated with an individual's experience, knowledge, skills, attitudes, and behavior. In this systematic review, the most cited individual level barrier was conflicting life choices $(n=38)$. Some examples include student-parents experiences 
balancing multiple roles as parents, students and employees, time demands, career compromise, and academic vulnerability. In addition, financial barriers $(n=30)$ also impede student-parent's ability to afford childcare services, school tuitions, and quality housing. Another barrier reported was student-parent's self-perceptions about self-doubt, cognitive dissonance, negative perception about welfare programs, sexism, and perfectionism $(n=17)$. Next, poor mental health $(n=16)$, including postpartum depression, stress, feelings of isolation, and marginalization, was reported. Further, poor knowledge about available campus resources and programs were also cited as barriers $(n=9)$. Finally, international students found it challenging to blend into a new society because of language barriers and cultural variations $(n=3)$.

\subsubsection{Interpersonal-level factors}

Interpersonal-level factors comprise interactions with formal and informal social networks and social support systems. This study investigated the relationship between studentparents and faculty/staff, family members, and peers. Student-parent's relationship with faculty and staff was the greatest barrier reported in this study $(n=13)$. These barriers were related to misaligned relationships, poor communication, and lack of understanding of student-parents ordeals. Faculty/staff's unwillingness to allow student-parents to bring their children to class and the denial of employment opportunities because of their parental status were barriers reported.

Additionally, student-parents said poor social relationships with peers (i.e., non-studentparents) posed challenges to their educational attainment $(n=10)$. These include uncooperative peers during group tasks and unwelcoming attitudes from the student population about breastfeeding on campus. Lastly, poor relationships with family members also posed challenges to student-parents educational pursuit $(n=9)$. These challenges include unsupportive partners' 
reluctance to watch over their children while their partners studied, the death of family members, and family problems such as broken relationships.

\subsubsection{Institutional-level factors}

Institutional or organizational level factors include policies, programs, and regulations for operation. In this study, we investigated factors within HE. Of the barriers identified, institutional policies $(n=41)$ were the most reported challenges to student-parents' academic outcomes. Within this category, lack of family-friendly events, university-age policies on children, unaffordable/lack of on-campus childcare services, lack of campus-based resources, and insufficient financial aid. Scheduling conflicts $(n=10)$ relating to class scheduling conflicts with on-campus childcare policies, hindering students' ability to attend classes. This made students miss class or adjust their class schedules to mirror their children's school schedule. Finally, the lack of a comprehensive student-parent database $(n=2)$ was noted.

\subsubsection{Policy-level factors}

Policy-level factors include local, state, and national policies and laws. In this study, two key themes emerged. First, international students on F1 or J1 visas reported that immigration policies $(n=1)$ restricting their spouses from earning an income reduced their earning power and made it challenging for them to manage their home dynamics. Students also reported that the Free Application for Federal Student Aid (FAFSA) documentation process made it difficult to complete their application $(n=1)$.

Table 2: Barriers of Student Parents' Academic Success in IHE in the US Reported Using the Socioecological Model

Socio-ecological levels

Individual-level factors

Financial issues

Childcare

Housing

\section{n Study ID numbers}

30

$1,8,9,16,19,26,29,37$

$1,16,26,29,37$ 
Schoolfees

Low income

Conflicting life choices

Balancing school, work, family

Time demands

Career compromise

Academic vulnerability

\section{Self-perception}

Self-doubt

Cognitive dissonance/Identity

Perfectionism

Racism, sexism, classism

Negative perception about welfare programs

\section{Mental Health}

Feelings of isolation

Stress, depression

Marginalization

Acculturation

Adjustment to living in two cultures

Language difficulty

Campus resources

Unaware of campus resources

Underutilization of campus

resources

Transportation challenges

\section{Interpersonal-level factors}

Faculty and staff

Attitudes and support

\section{Family}

Abusive and unstable relationships with partners

Death of family member/friend

Peer support

Poor social support
$1,16,19,26,29,32,37$

$1,2,5,6,10,12,22,26,28,38$

38

$2,4,8,9,10,13,14,15,16,18,19,20,21,23,25,26,29,31,32,33,38$, 41

$3,4,19,25,31,32,34,35$

$4,5,28,32$

$2,6,11,19$

17

$7,21,32,38$

$13,24,39$

13

$7,24,37,38$

$1,2,7,12,38$

16

$16,20,24,38$

$9,6,21,26,33,36,31$

$7,20,29,32,39$

3

16,24

16

9

$3,5,8,9,34,36,37,40$

3

$419,26,37,40$

13

$1,3,5,9,14,19,20,24,31,32,34,37,39$

9

$2,4,6,9,16,20,21$

5,6

10

$4,9,13,14,16,20,22,24,31,34$

\section{Institutional-level factors}




\begin{tabular}{|c|c|c|}
\hline University policies & 41 & \\
\hline Lack of family friendly events & & $1,8,9,19,16,20,25,31,34$ \\
\hline $\begin{array}{l}\text { University age limit policies on } \\
\text { children }\end{array}$ & & $1,8,25$ \\
\hline $\begin{array}{l}\text { Unaffordable/lack of Childcare } \\
\text { services }\end{array}$ & & $1,3,8,9,16,17,19,23,25,26,28,29,37$ \\
\hline Lack of campus-based resources & & $1,5,8,9,16,17,21,23,28,31,34,37$ \\
\hline Financial aid & & $8,19,37,40$ \\
\hline Scheduling & 10 & \\
\hline $\begin{array}{l}K-12 \text { and university scheduling } \\
\text { conflicts }\end{array}$ & & $1,19,21,25,26,31,32,34,37,40$ \\
\hline Parenting student database & 2 & 1,40 \\
\hline Public policy-level factors & 2 & \\
\hline Restrictive immigration policies & 1 & 24 \\
\hline Low levels of FAFSA completion & 1 & 11 \\
\hline
\end{tabular}

Note: Study ID Numbers corresponds with articles in all Tables.

\subsection{Secondary Theme: Facilitators to Student-parents' Academic Outcomes}

Table 3 shows that facilitators to student-parents academic outcomes. The most facilitators to student-parents academic success were within the individual level $(n=51)$. These included student-parents personal experiences, personal goals, self-awareness, self-efficacy, and spirituality that enabled them to navigate the school environment and the barriers they encounter. Interpersonal level factors ( $\mathrm{n}=34$ ) such as supportive advising from faculty and staff, strong bond with family members, flexible work schedule, and strong social support from peers facilitated student-parents academic success. University policies $(n=32)$ such as affordable on-campus childcare centers, campus-based support programs, family-oriented campus environment, and flexible class deadlines emerged as themes that helped student-parents navigate the challenges they encounter. Lastly, public policy $(n=7)$ related to welfare programs such as federal income programs and financial aid were reported as facilitators to student-parents academic pursuits. 
Table 3: Facilitators of Student Parents' Academic Success in IHE in the US Reported Using the Socioecological Model

\begin{tabular}{l} 
Socio-ecological levels \\
\hline Individual \\
Personal goals \\
Future financial autonomy \\
Motivation as role models for children/to attend \\
college \\
Experience \\
Prior knowledge, discipline and experience \\
Help-seeking skills \\
Safety nets (emergency cash, place to live, childcare)
\end{tabular}

\section{Self-awareness}

Self-confidence/awareness/identity

Positive mindset

\section{Self-efficacy}

Compartmentalize issues with cognitive dissonance

Goal setting

19

n Study ID numbers

Time management/scheduling

Intrinsic/Extrinsic motivational orientation

\section{Spirituality}

\section{Interpersonal}

\section{Faculty}

13

Faculty support

Culturally responsive advising

\section{Family}

Family support

\section{Employer}

Flexible work schedule

Peer support

Social support

\section{Institutional}

\section{Policies}

21

Campus-based support agents/counselling service Access to childcare

$3,12,4,25,4,21,32,41$

$2,3,4,9,10,12,25,26,29,31,32,41$

13

$18,31,14,21,32,41$

$8,9,25,26,31$

4,12

7

$4,14,26,32,39$

14,26

12

13

14,26

$10,14,18,25,26$

$10,14,29,31$

126

$4,5,8,14,18,26,29,30,32,37,39$

8,30

12

$3,4,9,12,14,18,26,27,29,31,33,34$

1

18

8

$8,12,14,21,31,30,34,38$

$3,8,23,25,29,35$

$8,9,5,23,29,32$ 
Paid parental leave

Health insurance for dependents

Financial support/scholarship

Family oriented campus environment

Family housing facilitates

Enhancing family-friendly university culture

Mentoring, faculty training

Scheduling

Extension of deadlines and part-time options

Project-based learning environments

\section{Public policy}

Welfare programs

Federal income programs (Food stamp)

Financial aid
5

5

$14,23,26,32,37,38,40$

7

1 ,

$5,8,32$

3

$5,17,32$

122

7

$14,11,26$

Note: Study ID Numbers correspond with articles in all Tables.

\subsection{Evidence of programs and policies for student-parents}

Of the 41 studies included, only 10 reported institutional efforts to enhance the studentparent's academic experience. These efforts ranged from on-campus childcare centers, studentparents social community programs, and designated lactation rooms for breastfeeding studentmothers. In unison, these studies noted that these programs were necessary to ease studentparents burden and promote a more inclusive atmosphere within the university setting -Table 4 .

The most cited recommendation from these articles for facilitating student parents' study was providing on-campus childcare and designated breastfeeding areas. Additional

recommendations include support groups that involve other student parents and faculty and staff, clear university policies tailored to student parents, and public policy supports that enhance the student parents' access to programs that are focused on limiting barriers to higher education for this population. A combination of these recommendations could prove useful for addressing the educational needs of parenting students. 
Table 4: Studies Reporting Evidence Existing of Programs or Policies for Pregnant and Parenting Students

\begin{tabular}{|c|c|c|}
\hline ID & Author/year & Services provided \\
\hline 1 & (Yakaboski, 2010) & $\begin{array}{l}\text { The institution offered family housing facilities as a part of the } \\
\text { residence life departments }\end{array}$ \\
\hline 8 & (Sallee \& Cox, 2019) & $\begin{array}{l}\text { On-campus childcare center and a unit with the student service } \\
\text { division serves students with children }\end{array}$ \\
\hline 9 & (Roy et al., 2018) & Participants were recruited from an on-campus childcare center \\
\hline 16 & (Myers-Walls et al., 2011) & $\begin{array}{l}\text { Participants were recruited from on-campus housing for } \\
\text { married graduate students }\end{array}$ \\
\hline 17 & (Molter et al., 2019) & Evidence of pregnancy and lactation guidelines \\
\hline 29 & (Kensinger \& Minnick, 2018) & $\begin{array}{l}\text { Nontraditional student organization, on-campus child and } \\
\text { family center, and a Head Start Program located on campus. }\end{array}$ \\
\hline 31 & (Hotez et al., 2020) & A student-parent task force \\
\hline 34 & (Dinour et al., 2015) & $\begin{array}{l}\text { One lactation room on this campus, available Monday through } \\
\text { Friday from 8:30 am to 4:30 pm upon appointment. }\end{array}$ \\
\hline 39 & (Auguste et al., 2018) & Nontraditional student community \\
\hline 40 & (Askelson et al., 2020) & $\begin{array}{l}\text { A one-year on-campus federal Pregnancy Assistance Funded } \\
\text { (PAF) program implemented for pregnant and parenting } \\
\text { students }\end{array}$ \\
\hline
\end{tabular}

Note: Study ID Numbers correspond with articles in all Tables.

\subsection{Methodological quality}

\subsubsection{Theoretical framework}

Most qualitative studies reported at least one theoretical framework $(n=16)$ than the quantitative studies $(n=5)$. The theories utilized in this Study predominantly varied. The critical feminist theory $(n=4)$ was the most utilized framework, followed by the ecological model $(n=2)$.

\subsubsection{Risk of bias}

Concerning the risk of bias, all studies met at least five quality evaluation tools for quantitative and qualitative assessment. We could not precisely rank the studies on some of the tool's quality attributes among the reviews. However, the overall quality of the studies was high. The qualitative studies' sources of bias were 1) evidence of ethical approval by an appropriate 
body or 2) the researcher's influence and vice-versa (reflexivity). In terms of the quantitative studies, sources of bias were predominantly related to the statistical analysis used. Detailed information is seen in Tables 5 and 6.

Table 5: Methodological Quality of the Qualitative Articles Using the Joanna Briggs Critical Appraisal Checklist for Qualitative Research

\begin{tabular}{lllllllllllll}
\hline ID & Author Year & \multicolumn{1}{c}{ JBI Critical Appraisal Attributes* } \\
& & Q1 & Q2 & Q3 & Q4 & Q5 & Q6 & Q7 & Q8 & Q9 & Q10 \\
\hline 1 & (Yakaboski, 2010) & + & + & + & + & + & + & - & + & + & + \\
2 & (Wilson \& Cox, 2011) & + & + & + & + & + & + & - & + & - & + \\
4 & (Deutsch \& Schmertz, 2011) & + & + & + & + & + & + & + & + & - & + \\
7 & (Sealey-Ruiz, 2013) & + & + & + & + & + & + & + & + & - & + \\
8 & (Sallee \& Cox, 2019) & + & + & + & + & + & + & - & + & - & + \\
9 & (Roy et al., 2018) & - & + & + & + & + & - & - & + & - & + \\
13 & (Prikhidko \& Haynes, 2018) & + & + & + & + & + & + & + & + & + & + \\
14 & (Peterson, 2016) & + & + & + & + & + & + & + & + & + & + \\
15 & (Onwuegbuzie et al., 2014) & + & + & + & + & + & + & + & + & - & + \\
16 & (Myers-Walls et al., 2011) & + & + & + & + & + & + & + & + & - & + \\
18 & (Sallee, 2015) & + & + & + & + & + & + & - & + & - & + \\
20 & (Madden, 2018) & + & + & + & + & + & + & + & + & - & + \\
21 & (Shepherd \& Nelson, 2012) & + & + & + & + & + & - & - & + & + & + \\
24 & (Lobnibe, 2013) & + & + & + & + & + & + & - & + & - & + \\
25 & (Lindsay \& Gillum, 2018) & + & + & + & + & + & + & - & + & - & + \\
26 & (Lashley \& Ollivierre, 2014) & + & + & + & + & + & - & - & + & + & + \\
29 & (Kensinger \& Minnick, 2018) & + & + & + & + & + & + & + & + & + & + \\
31 & (Hotez et al., 2020) & - & + & + & + & + & - & - & + & - & + \\
32 & (Markle, 2015) & + & + & + & + & + & + & - & + & + & + \\
33 & (Eisenbach, 2013) & - & + & + & + & + & - & - & + & - & + \\
34 & (Dinour et al., 2015) & + & + & + & + & + & + & - & + & - & + \\
37 & (Brown \& Nichols, 2013) & + & + & + & + & + & + & + & + & - & + \\
38 & (Bloom, 2009) & - & + & + & + & + & - & + & + & - & + \\
39 & (Auguste et al., 2018) & + & + & + & + & + & - & - & + & - & + \\
40 & (Askelson et al., 2020) & - & + & + & + & + & - & - & + & + & + \\
41 & (E. D. n. Lovell, 2014) & - & + & + & + & + & - & - & - & - & + \\
\hline
\end{tabular}

Note: Study ID Numbers corresponds with articles in all Tables

JBI Critical Appraisal Attributes *

Q1 Is there congruity between the stated philosophical perspective and the research methodology? 
Q2 Is there congruity between the research methodology and the research question or objectives?

Q3 Is there congruity between the research methodology and the methods used to collect data?

Q4 Is there congruity between the research methodology and the representation and analysis of data?

Q5 Is there congruity between the research methodology and the interpretation of results?

Q6 Is there a statement locating the researcher culturally or theoretically?

Q7 Is the influence of the researcher on the research, and vice- versa, addressed?

Q8 Are participants, and their voices, adequately represented?

Q9 Is the research ethical according to current criteria or, for recent studies, and is there evidence of ethical approval by an appropriate body?

Q10 Do the conclusions drawn in the research report flow from the analysis, or interpretation, of the data?

Table 6: Methodological Quality of The Quantitative Articles Using the Joanna Briggs Critical Appraisal Checklist for Cross-sectional Studies

\begin{tabular}{llllllllll}
\hline ID & Author Year & QB & Q2 & Q3 & Q4 & Q5 & Q6 & Q7 & Q8 \\
\hline 3 & (Wilsey, 2013) & + & + & - & + & + & + & + & - \\
5 & (Springer et al., 2009) & + & + & + & + & - & - & + & - \\
6 & (Shenoy et al., 2016) & + & + & + & + & - & + & + & + \\
10 & (Ricco et al., 2009) & + & + & + & + & + & + & + & + \\
11 & (Radey \& Cheatham, 2013) & + & + & + & + & + & + & + & + \\
12 & (Radey, 2017) & + & + & + & + & + & + & + & + \\
17 & (Molter et al., 2019) & + & + & + & + & + & + & + & + \\
19 & (Mahaffey \& Gregg, 2015) & + & + & + & + & + & - & + & - \\
22 & (E. Lovell \& Munn, 2017) & + & + & + & + & + & + & + & + \\
23 & (E. D. N. Lovell, 2014) & + & + & + & + & + & + & + & + \\
27 & (Plageman \& Sabina, 2010) & + & + & + & + & + & + & + & - \\
28 & (Kulp, 2020) & + & + & + & + & + & + & + & + \\
30 & (Johnson \& Nussbaum, 2012) & + & + & + & + & + & + & + & + \\
35 & (Crispin \& Nikolaou, 2019) & + & + & - & - & + & + & + & + \\
36 & (Bye et al., 2017) & + & + & - & - & + & + & + & - \\
\hline
\end{tabular}

Note: Study ID Numbers corresponds with articles in all Tables

JBI Critical Appraisal Attributes *

Q1 Were the criteria for inclusion in the sample clearly defined?

Q2 Were the study subjects and the setting described in detail? 
Q3 Was the exposure measured in a valid and reliable way?

Q4 Were objective, standard criteria used for measurement of the condition?

Q5 Were confounding factors identified?

Q6 Were strategies to deal with confounding factors stated?

Q7 Were the outcomes measured in a valid and reliable way?

Q8 Was appropriate statistical analysis used?

\begin{tabular}{lc}
\hline JBI Critical Appraisal Key & \\
\hline Low risk of bias & + \\
High and unclear risk of bias & - \\
\hline
\end{tabular}

\section{Discussions}

This study is the first to summarize student-parents experiences in HE in the United States systematically. In this study, we used the SEM to delineate the predictors of academic success among student-parents. Our study showed that the barriers to student-parents educational success are a systems problem that necessitates multilevel intervention and strategies across several settings (Lin, 2016; Osam et al., 2017; Quaye et al., 2019). Importantly, this review revealed that a single-level approach (e.g., access to on-campus childcare centers only) to meeting student-parents diverse needs is not feasible. Results from this study have direct implications for policy, school administrators, educational researchers, and students.

Our result showed that HE is a significant leverage point to address the barriers and improve student-parents academic outcomes as institutional policies were major predictors of student-parents academic success. This result is not surprising because HE is a complex system with several elements (e.g., administrators, faculty, and staff) that interact to predict academic outcomes (Quaye et al., 2019). For example, institutional policies (e.g., family-friendly policies, on-campus childcare centers, campus-based resources, financial aid) were significant barriers reported. Yet, relationships with faculty and staff were also cited as barriers to student-parents 
academic outcomes predicated in part by institutional policies. As such, HE should prioritize creating an inclusive campus environment that attends to the needs of student-parents.

This review highlights the importance of expanding access to diverse funding opportunities for student-parents. Addressing financial problems requires intervention specifically at the macro-level (i.e., HE). One such approach is for HE to guide students through the complexities and documentation of the financial aid (e.g., filing FAFSA and Pell Grant) applications process. HE can leverage other opportunities such as revising the cost of attendance and including the number and ages of dependent children on the FAFSA (Wladis et al., 2018). Additionally, collaborating with community partners should be encouraged. Programs such as Aspen Ascend Two-Generation through their Aspen Postsecondary Success for Parent Initiative (The Aspen Institute, 2020) are a potential resource HE can leverage. In 2020, the initiative instituted a student-led fund supporting organizations and institutions that support studentparents postsecondary success.

Another approach is to tap into existing federal benefit programs that provide childcare access to low-income families, such as CCAMPIS, Child Care and Development Funds (CCDF), Temporary Assistance for Needy Families (TANF), and Head Start (United States Government Accountability Office, 2019). We applaud efforts made by the institution reported in (Askelson et al., 2020) in their use of the Pregnancy Assistance Funded (PAF) to create an inclusive campus climate for student-parents. Importantly, HE needs to develop internal pathways to support their student-parent population. The City University of New York (CUNY), Accelerated Study in Associate Programs (ASAP), is a success story that can be replicated and modified through a student-parent lens (Levin et al., 2018). 
In this review, most studies focused on the individual-level factors that affect studentparents educational outcomes. In contrast, those reporting other factors within the interpersonal, institutional, and policy factors were limited. Since factors that predict student-parents outcomes are diverse, more research is needed to access and evaluate policy-level factors such as federal aid programs and their effectiveness in meeting student-parents needs.

Furthermore, although most of the articles reported that students encountered significant challenges balancing their multiple roles and conflicting time demands, recommendations on managing these challenges were not reported save for only two articles that encouraged students to self-advocate. It is important that researchers examine coping strategies that may help studentparents navigate their intersecting roles to achieve academic success. Coping can determine whether a stressful event leads to adaptive or maladaptive outcomes (Dardas \& Ahmad, 2015). This review indicates that by identifying coping strategies, student-parents can take full responsibility for their situations, enabling them to find more meaning in their educational journey. To this end, providing student-parents with the needed skills and support services to allow them to cope with their responsibilities to improve their academic outcomes is imperative. Other noteworthy individual-level factors for future research include motivations, persistence, and self-efficacy.

This review showed that challenges within the individual (e.g., perfectionism), intrapersonal (e.g., poor relationships with family members), institutional (e.g., lack of familyfriendly programs), and policy (e.g., immigration policies) interplay to adversely affect mental health. Importantly, this study revealed that research into the intersection of student-parents and mental health is scant. Research shows that depression and anxiety are prevalent among college students so as in parents (Hefner \& Eisenberg, 2009; McLeish \& Redshaw, 2017; National 
Alliance on Mental Illness, 2012). Among mothers in an Early Head Start program, those with depression did not increase their participation in educational activities, employment, or job training (Schmit \& Walker, 2016). Therefore, future studies should examine the factors such as social support and mental health services within the school system.

Furthermore, future research should investigate the role of mediating and moderating factors (e.g., international student status) on student-parent outcomes. Only two articles reported the experiences of international students who are parents in HE. A systematic review of international students' psychological adjustment to life in the United States showed that these students undergo divergent barriers that adversely affect their psychological wellbeing (Zhang \& Goodson, 2011). As such, international students who are parents may be doubly impacted by their diverse intersections.

Lastly, regarding methodological quality, most of the reviewed studies met the Joanna Briggs Institute (JBI) Critical appraisal criteria, but there is more room for improvement. Very few qualitative studies reported ethical approval. Researchers should strengthen their studies by ensuring they report how research subjects are protected. Second, quantitative researchers should utilize robust statistical analysis to provide precise estimates of their findings to ensure replicability and generalizability.

\subsection{Implications for practice}

The findings in this review go beyond closing educational gaps but transcend into equity issues and social justice. Considering that most students who are parents are historically from disadvantaged backgrounds, as stated earlier in this manuscript, attending to their needs is beneficial to the student themselves, future generations, and society. This review shows no single solution to addressing student-parents educational needs; rather, understanding the multilevel 
components that interplay with each other to predict student-parents' academic success is important. This review's findings can inform relevant stakeholders into creating an inclusive campus environment for student-parents in HE. To improve student-parents academic success, stakeholders need to address multilevel barriers and intervene at a specific SEM level summarized in this review. Similarly, this review can inform student-parents of the multiple factors that may influence their academic success. Specifically, students may focus on relevant SEM factors and make adjustments to facilitate academic success.

\subsection{Limitations}

Although this review adds to the literature, this systematic review had several limitations. First, we only reviewed studies conducted in the United States, so the results are not generalizable beyond the US. Also, most of the studies employed qualitative methodology, which may limit their generalizability. However, this concern is negligible because results from the longitudinal and cross-sectional studies in this review corroborate with the results from the qualitative analysis. Moreover, some of the articles analyzed comparison groups that provided a robust understanding of the differential impact of parenting status on academic outcomes. Thirdly, we excluded a considerable number of dissertations, which may contain additional information not captured in the articles reviewed. But it is unlikely that we would have found new themes. We conducted a robust search, and the articles reviewed have undergone the peerreview process, which strengthens the studies' methodological qualities.

\subsection{Conclusion}

The current study explored the lived experiences of students who are parents - most of whom were mothers - in HE in the United States. The link between parents' education status, their children's educational and future aspirations, economic stability, and overall health is well 
documented, as is the impact on society. This review shows that improving student-parents academic outcomes transcend beyond just academic success for the parent and into equity issues. As such, closing gaps in educational outcomes should be a top priority for institutions and policymakers.

Funding: None

\section{Author Contribution}

K.V.A: Conceptualization, Methodology, Formal analysis, Investigation, Data curation, Writing - original draft, Writing - review \& editing, Visualization. G.O: Methodology, Formal analysis, Investigation, Data curation, Writing - original draft, Writing - review \& editing, Visualization. S.P: Methodology, Formal analysis, Investigation, Data curation, Writing - original draft, Writing - review \& editing. O.A: Methodology, Formal analysis, Investigation, Data curation. W.G: Methodology, Data curation, Writing - review \& editing. L.J.M: Methodology, Formal analysis, Investigation, Writing - review \& editing

Declaration of competing interest: None.

\section{Acknowledgments:}

We would like to thank [blinded for peer review] for their help with conducting the literature search. 
References

Askelson Natoshia, Ryan Grace, Pieper Felicia, Bash-Brooks Whitney, Rasmusson Addie,

Greene Mary, B. A. (2020). Perspectives on Implementation: Challenges and Successes of a Program Designed to Support Expectant and Parenting Community College Students in Rural, Midwestern State.

Auguste, E., Packard, B. W.-L., \& Keep, A. (2018). Nontraditional Women Students' Experiences of Identity Recognition and Marginalization During Advising. NACADA Journal, 38(2), 45-60. https://doi.org/10.12930/nacada-17-046

Bloom, L. R. (2009). "When one person makes it, we all make it": A study of Beyond Welfare, a women-centered community-based organization that helps low-income mothers achieve personal and academic success. International Journal of Qualitative Studies in Education, 22(4), 485-503. https://doi.org/10.1080/09518390902740597

Brown, V., \& Nichols, T. R. (2013). Pregnant and Parenting Students on Campus: Policy and Program Implications for a Growing Population. Educational Policy, 27(3), 499-530. https://doi.org/10.1177/0895904812453995

Bureau, U. S. C. (2018). More Than 76 Million Students Enrolled in US Schools, Census Bureau Reports. https://www.census.gov/newsroom/press-releases/2018/school-enrollment.html

Bye, E. M., Brisk, B. W., Reuter, S. D., Hansen, K. A., \& Nettleman, M. D. (2017). Pregnancy and Parenthood During Medical School. South Dakota Medicine: The Journal of the South Dakota State Medical Association, 70(12), 551-555. 
Crispin, L., \& Nikolaou, D. (2019). Balancing college and kids: estimating time allocation differences for college students with and without children. Monthly Labor Review, February, 1-12. https://doi.org/10.21916/mlr.2019.3

Cruise, R. Lindsey; Holtzman, Tessa; Gault, Barbara; Croom, David; Polk, P. (2019). Parents in College By the Numbers. https://iwpr.org/iwpr-issues/student-parent-successinitiative/parents-in-college-by-the-numbers/

Cruse, L.R., Holtzman, T., Gault, B., Croom, D., \& Polk, P. (2019). Parents in College By the Numbers. https://iwpr.org/iwpr-issues/student-parent-success-initiative/parents-in-collegeby-the-numbers/

Dardas, L. A., \& Ahmad, M. M. (2015). Coping strategies as mediators and moderators between stress and quality of life among parents of children with autistic disorder. Stress and Health, 31(1), 5-12.

Dammhahn, M., \& Kappeler, P. M. (2009). Females go where the food is: Does the socioecological model explain variation in social organisation of solitary foragers? Behavioral Ecology and Sociobiology, 63(6), 939-952. https://doi.org/10.1007/s00265-009-0737-2

Deutsch, N. L., \& Schmertz, B. (2011). “Starting from ground zero:” Constraints and experiences of adult women returning to college. Review of Higher Education, 34(3), 477504. https://doi.org/10.1353/rhe.2011.0002

Dinour, L. M., Pope, G. A., \& Bai, Y. K. (2015). Breast milk pumping beliefs, supports, and barriers on a university campus. Journal of Human Lactation, 31(1), 156-165. https://doi.org/10.1177/0890334414557522 
Eisenbach, B. (2013). Finding a balance: A narrative inquiry into motherhood and the doctoral process. Qualitative Report, 18(17), 1-13.

Figueiredo, J., \& Pereira, H. M. (2011). Regime shifts in a socio-ecological model of farmland abandonment. Landscape Ecology, 26(5), 737-749. https://doi.org/10.1007/s10980-0119605-3

Garney, W., Wilson, K., Ajayi, K. V., Panjwani, S., Love, S. M., Flores, S., ... \& Esquivel, C. (2021). Social-Ecological Barriers to Access to Healthcare for Adolescents: A Scoping Review. International Journal of Environmental Research and Public Health, 18(8), 4138.

Garrard, J. (2020). Health sciences literature review made easy. (6th ed.).

Gault, Barbara, Reichlin, Lindsey; Reynolds, Elizabeth; Froehner, M. (2014). Student Parents' Access to Campus Child Care Continued to Decline in 2015. IWPR \#C425. Student Parents’ Access to Campus Child Care Continued to Decline in 2015

Geiger, A.W.; Livingston, Gretchen., Bialik, K. (2019). 6 facts about US moms. https://www.pewresearch.org/fact-tank/2019/05/08/facts-about-u-s-mothers/

Gökçay, G. (2010). Breastfeeding and child cognitive development. Child: Care, Health and Development, 36(4), 591. https://doi.org/10.1111/j.1365-2214.2009.01070.x

Hefner, J., \& Eisenberg, D. (2009). Social Support and Mental Health Among College Students. 79(4), 491-499.

Hotez, E., Lin, H., Chan, V., Felix, J., Francis, A., Giacinto, D., Mitchell, G., \& Siddique, F. (2020). “If I Spent Five Hours Giving Birth Then I Can Do This Final:” A Qualitative 
Investigation of College Students With Children. Translational Issues in Psychological Science, 6(2), 147-159. https://doi.org/10.1037/tps0000230.supp

Johnson, M. L., \& Michael Nussbaum, E. (2012). Achievement goals and coping strategies: Identifying the traditional/nontraditional students who use them. Journal of College Student Development, 53(1), 41-54. https://doi.org/10.1353/csd.2012.0002

Kensinger, C., \& Minnick, D. J. (2018). The Invisible Village: An Exploration of Undergraduate Student Mothers' Experiences. Journal of Family and Economic Issues, 39(1), 132-144. https://doi.org/10.1007/s10834-017-9535-6

Kulp, A. M. (2020). Parenting on the Path to the Professoriate: A Focus on Graduate Student Mothers. Research in Higher Education, 61(3), 408-429. https://doi.org/10.1007/s11162019-09561-Z

Lashley, M.-B., \& Ollivierre, L. (2014). Exploring The Linkages Of Single Black Mothers And Their College Experiences. Global Education Journal, 138-155. http://content.ebscohost.com/ContentServer.asp?T=P\&P=AN\&K=99284197\&S=R\&D=ehh \&EbscoContent=dGJyMMTo50SeprM4zdnyOLCmr1CeqLFSs664SLOWxWXS\&Content Customer=dGJyMPGtsUuyr7NQuePfgeyx44Dt6fIA

Levin, H. M., \& García, E. (2018). Accelerating Community College Graduation Rates : A Benefit - Cost Analysis Accelerating Community College Graduation Rates : A Benefit Cost Analysis. The Journal of Higher Education, 89(1), 1-27. https://doi.org/10.1080/00221546.2017.1313087

Lim, S. J. J., \& Hoot, J. L. (2015). Bullying in an increasingly diverse school population: A 
socio-ecological model analysis. School Psychology International, 36(3), 268-282.

https://doi.org/10.1177/0143034315571158

Lin, X. (2016). Barriers and Challenges of Female Adult Students Enrolled in Higher Education: A Literature Review. Higher Education Studies, 6(2), 119. https://doi.org/10.5539/hes.v6n2p119

Lindsay, T. N., \& Gillum, N. L. B. (2018). Exploring Single-Mother College Students' Perceptions of Their College-Related Experiences and of Campus Services. Journal of Continuing Higher Education, 66(3), 188-199. https://doi.org/10.1080/07377363.2018.1537657

Lobnibe, J.-F. Y. (2013). Different Worlds, Mutual Expectations: African Graduate Student Mothers and the Burden of US Higher Education. Journal of Education and Learning, 2(2), 201-209. https://doi.org/10.5539/jel.v2n2p201

Lovell, E. D. n. (2014). Female College Students Who Are Parents: Motivation Clarified by the Ages of Their Children. Community College Journal of Research and Practice, 38(4), 370374. https://doi.org/10.1080/10668926.2013.780002

Lovell, E. D. N. (2014). College students who are parents need equitable services for retention. Journal of College Student Retention: Research, Theory and Practice, 16(2), 187-202. https://doi.org/10.2190/CS.16.2.b

Lovell, E., \& Munn, N. (2017). Introduction to Psychology Students' Parental Status Predicts Learning Preferences and Life Meaning. Journal of The First-Year Experience \& Students in Transition, 29(1), 119-136. 
Madden, M. (2018). Illuminating Low-income Pregnant and Parenting Student Mothers' Experiences with Community College. Equity and Excellence in Education, 51(3-4), 378395. https://doi.org/10.1080/10665684.2019.1571463

Mahaffey A. Barbara, Hungerford Gregg, S. S. (2015). College Student Mother Needs at Regional Campuses: An Exploratory Study. Journal of Chemical Information and Modeling, 01(01), 1689-1699.

Markle, G. (2015). Factors Influencing Persistence Among Nontraditional University Students. Adult Education Quarterly, 65(3), 267-285. https://doi.org/10.1177/0741713615583085

McLeish, J., \& Redshaw, M. (2017). Mothers' accounts of the impact on emotional wellbeing of organised peer support in pregnancy and early parenthood: A qualitative study. $B M C$ Pregnancy and Childbirth, 17(1), 1-14. https://doi.org/10.1186/s12884-017-1220-0

Mcleroy, K. R., Bibeau, D., Steckler, A., \& Glanz, K. (1988). An Ecological Perspective on Health Promotion Programs. Health Education \& Behavior, 15(4), 351-377. https://doi.org/10.1177/109019818801500401

Molter, B., Wayne, A., Mueller, M. K., Gibeley, M., \& Rosenbaum, M. H. (2019). Current policies and support services for pregnant and parenting veterinary medical students and house officers at United Statesveterinary medical training institutions. Journal of Veterinary Medical Education, 46(2), 145-152. https://doi.org/10.3138/jvme.0917-119r

Munn, Z., Peters, M. D. J., Stern, C., Tufanaru, C., McArthur, A., \& Aromataris, E. (2018). Systematic review or scoping review? Guidance for authors when choosing between a systematic or scoping review approach. BMC Medical Research Methodology, 18(1), 1-7. 
https://doi.org/10.1186/s12874-018-0611-X

Myers-Walls, J. A., Frias, L. V., Kwon, K. A., Ko, M. J. M., \& Lu, T. (2011). Living life in two worlds: Acculturative stress among Asian international graduate student parents and spouses. Journal of Comparative Family Studies, 42(4), 455-478. https://doi.org/10.3138/jcfs.42.4.455

National Alliance on Mental Illness. (2012). College Student Speaks. https://www.nami.org/Support-Education/Publications-Reports/Survey-Reports/CollegeStudents-Speak_A-Survey-Report-on-Mental-H

Oishi, S., Rothman, A. J., Snyder, M., Su, J., Zehm, K., Hertel, A. W., Gonzales, M. H., \& Sherman, G. D. (2007). The Socioecological Model of Procommunity Action: The Benefits of Residential Stability. Journal of Personality and Social Psychology, 93(5), 831-844. https://doi.org/10.1037/0022-3514.93.5.831

Onwuegbuzie, A. J., Rosli, R., Ingram, J. M., \& Frels, R. K. (2014). A critical dialectical pluralistic examination of the lived experience of select women doctoral students. Qualitative Report, 19(3).

Osam, E. K., Bergman, M., \& Cumberland, D. M. (2017). An Integrative Literature Review on the Barriers Impacting Adult Learners' Return to College. Adult Learning, 28(2), 54-60. https://doi.org/10.1177/1045159516658013

Peterson, S. (2016). Community college student-parents: priorities for persistence. Community College Journal of Research and Practice, 40(5), 370-384. https://doi.org/10.1080/10668926.2015.1065210 
Pew Research Center. (2017). Gender and caregiving. https://www.pewsocialtrends.org/2017/03/23/gender-and-caregiving/

Plageman, P. M., \& Sabina, C. (2010). Perceived family influence on undergraduate adult female students. Journal of Continuing Higher Education, 58(3), 156-166. https://doi.org/10.1080/07377363.2010.491768

Prikhidko, A., \& Haynes, C. (2018). Balancing graduate school and mothering: Is there a choice? International Journal of Doctoral Studies, 13, 313-326. https://doi.org/10.28945/4109

Quaye, S.J., Harper, S.R., \& Pendakur, S. L. (2019). Student Engagement in Higher Education: Theoretical perspectives and practical approaches for diverse populations (Eds). Routledge.

Radey, M. (2017). Postnatal college trajectories: When and under what circumstances do mothers enroll? Teachers College Record, 119(5).

Radey, M., \& Cheatham, L. P. (2013). Do single mothers take their share?: FAFSA completion among aid-eligible female students. Journal of Diversity in Higher Education, 6(4), 261275. https://doi.org/10.1037/a0035089

Ricco, R., Sabet, S., \& Clough, C. (2009). College mothers in the dual roles of student and parent: Implications for their children's attitudes toward school. Merrill-Palmer Quarterly, 55(1), 79-110.

Roy, R. N., Bradecich, A., Dayne, N., \& Luna, A. (2018). The Transition to Motherhood: The Experiences of College Student-Parents. Journal of Family \& Consumer Sciences, 110(3), 
48-57. https://doi.org/10.14307/jfcs110.3.48

Sallee, M. W. (2015). Adding academics to the work/family puzzle: Graduate student parents in higher education and student affairs. Journal of Student Affairs Research and Practice, 52(4), 401-413. https://doi.org/10.1080/19496591.2015.1083438

Sallee, M. W., \& Cox, R. D. (2019). Thinking beyond childcare: Supporting community college student-parents. American Journal of Education, 125(4), 621-645. https://doi.org/10.1086/704094

Schmit, Stephanie Walker, C. (2016). Seizing New Policy Opportunities to Help LowIncome Mothers with Depression: Current Landscape, Innovations, and Next Steps. https://www.clasp.org/sites/default/files/public/resources-and-publications/publication1/Opportunities-to-Help-Low-Income-Mothers-with-Depression-1-1.pdf

Sealey-Ruiz, Y. (2013). Learning to resist: Educational counter-narratives of black college reentry mothers. Teachers College Record, 115(4), 1-31.

Shenoy, D. P., Lee, C., \& Trieu, S. L. (2016). The Mental Health Status of Single-Parent Community College Students in California. Journal of American College Health, 64(2), 152-156. https://doi.org/10.1080/07448481.2015.1057147

Shepherd, J., \& Nelson, B. M. (2012). Balancing act: A phenomenological study of female adult learners who successfully persisted in graduate studies. Qualitative Report, 17(20), 1-21.

Springer W. Kristen, Parker K. Brenda, L.-R. C. (2009). Making Space for Graduate Student Parents Practice and politics. Nurse Practitioner, 29(1), 8. 
https://doi.org/10.1097/00006205-200401000-00003

The Aspen Institute. (2020). ASPEN INSTITUTE ANNOUNCES FIRST-EVER PARENT POWERED SOLUTIONS FUND GRANTEES.

The Lancet Public Health. (2020). Education: a neglected social determinant of health. The Lancet Public Health, 5(7), e361. https://doi.org/10.1016/S2468-2667(20)30144-4

Townsend, N., \& Foster, C. (2013). Developing and applying a socio-ecological model to the promotion of healthy eating in the school. Public Health Nutrition, 16(6), 1101-1108. https://doi.org/10.1017/S1368980011002655

US Department of Labor. (n.d.). Data and Statistics. https://www.dol.gov/agencies/wb/data

United States Census Bureau. (n.d.-a). Are Women Really Opting Out of Work After They Have Babies? Retrieved January 24, 2021, from https://www.census.gov/library/stories/2019/08/are-women-really-opting-out-of-work-afterthey-have-babies.html

United States Census Bureau. (n.d.-b). The Choices Working Mothers Make. Retrieved January 24, 2021, from https://www.census.gov/library/stories/2020/05/the-choices-workingmothers-make.html

United States Government Accountability Office. (2019). HIGHER EDUCATION More Information Could Help Student Parents Access Additional Federal Student Aid. https://www.gao.gov/assets/710/701002.pdf

Wilsey, S. A. (2013). Comparisons of adult and traditional college-age student mothers: Reasons 
for college enrollment and views of how enrollment affects children. Journal of College Student Development, 54(2), 209-214. https://doi.org/10.1353/csd.2013.0018

Wilson, K. B., \& Cox, E. M. (2011). No Kids Allowed: Transforming Community Colleges to Support Mothering. NASPA Journal About Women in Higher Education, 4(2). https://doi.org/10.2202/1940-7890.1077

Wladis, C., Hachey, A. C., \& Conway, K. (2018). No Time for College? An Investigation of Time Poverty and Parenthood. Journal of Higher Education, 89(6), 807-831. https://doi.org/10.1080/00221546.2018.1442983

Yakaboski, T. (2010). Going At It Alone: Single-Mother Undergraduate's Experiences. Journal of Student Affairs Research and Practice, 47(4), 463-481. https://doi.org/10.2202/19496605.6185

Zhang, J., \& Goodson, P. (2011). International Journal of Intercultural Relations Predictors of international students ' psychosocial adjustment to life in the United States: A systematic review. International Journal of Intercultural Relations, 35(2), 139-162. https://doi.org/10.1016/j.ijintrel.2010.11.011 